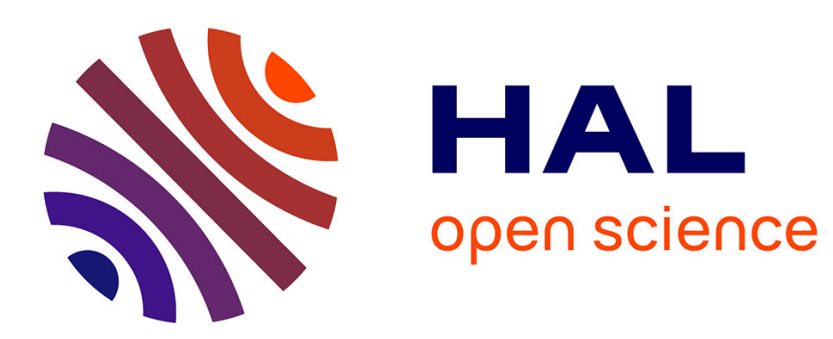

\title{
Autonomic dynamically reconfigurable architectures for future communication systems
}

\author{
Khalil Drira
}

\section{To cite this version:}

Khalil Drira. Autonomic dynamically reconfigurable architectures for future communication systems . The 9 th International Conference on Management of Digital EcoSystems (MEDES 2017), Nov 2017, Bangkok, Thailand. hal-01592617

\section{HAL Id: hal-01592617 https://hal.laas.fr/hal-01592617}

Submitted on 25 Sep 2017

HAL is a multi-disciplinary open access archive for the deposit and dissemination of scientific research documents, whether they are published or not. The documents may come from teaching and research institutions in France or abroad, or from public or private research centers.
L'archive ouverte pluridisciplinaire HAL, est destinée au dépôt et à la diffusion de documents scientifiques de niveau recherche, publiés ou non, émanant des établissements d'enseignement et de recherche français ou étrangers, des laboratoires publics ou privés. 


\title{
Autonomic dynamically reconfigurable architectures for future communication systems
}

\author{
[Invited Paper] \\ Khalil Drira \\ LAAS-CNRS, Université de Toulouse, CNRS \\ Toulouse \\ France \\ khalil@laas.fr
}

\begin{abstract}
Our objectives involve the development of correct by design models and operational solutions to discover, compose and manage, by automated procedures, the properties of adaptability essential for autonomous reconfiguration. These properties are critical for highly dynamic complex systems such as massive data collection, transport and processing systems, and associated IoT applications. This research direction is of interest for many applications that, on the one hand, have strong reconfiguration requirements to manage mobility, ubiquity, adaptation and self-healing, which are essential for autonomy, and that, on the other hand, are subject to non-functional constraints of quality of service, robustness and reliability. We propose, here, to develop multi-level reconfiguration solutions (VM clusters, application containers, message partitions), in order to manage simultaneously and consistently the adaptation in the different architectural levels. This is necessary for service continuity and end-to-end quality we implemented by coordinating the adaptation actions to satisfy the evolving of the context.
\end{abstract}

\section{CCS Concepts}

-Computer systems organization $\rightarrow$ n-tier architectures; •Software and its engineering $\rightarrow$ Software design engineering; •Networks $\rightarrow$ Cloud computing;

\section{Keywords}

adaptive systems; software architectures; dynamic reconfiguration

\section{INTRODUCTION}

\footnotetext{
${ }^{*}$ CNRS Research Director.
}

In recent years, the evolution of communication systems, accelerated by the Internet and its technologies, and the evolution of software and middleware technologies have resulted in the emergence of new distributed applications and have led to a new network landscape (Fig. 1). Applications are multi-media, multi-user, multi-component, and cooperatives. Networks have evolved into wireless communications, particularly ad-hoc. The types of devices and access points are now multiple and the services offered by the operators are generalized. Software and middleware technologies have evolved into service-oriented architectures.

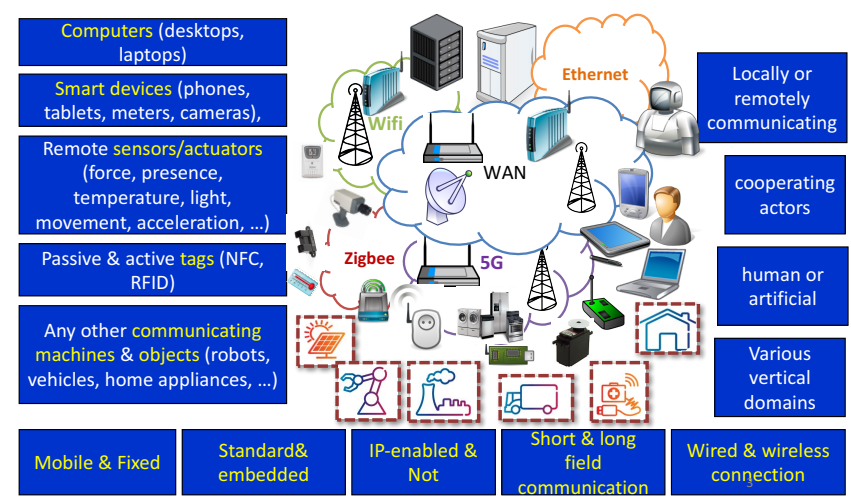

Figure 1: The new context of communication systems

The services and associated applications are now composed, deployed, and dynamically adapted. This composition includes the discovery and coordination of components, services and resources at the middleware and network levels. Components, implemented as microservices, services or software components, and the elements of the underlying network are brought to cooperate, horizontally and vertically, in a coordinated way to manage (i.e. monitor, analyze, repair) the quality of service required by applications and their users (human or artificial). Support for ubiquity and mobility, cooperation and group communication is one of the horizons of these advances. Actors can be artificial (robots, machines) or human (doctors, engineers, administrators, etc.) [2].

Within this horizon, our contributions have a double context (Fig. 2):

- an extended application context, mobile and scalable 
by its applications and use cases: cooperative applications mobile embedded and ubiquitous; use of different types of terminals and access points, cooperation spontaneous, and QoS requirements that are different from one case of application and use to another, ...

- a heterogeneous, open and variable-resource communication context, bringing together software with complex architectures, with no control on the number of participants in the cooperation activities supported. The resources correspond to servers and routers as well as the bandwidth of interconnection links.

Within this objective, and with regard to the architectural components, our effort focuses on the development of procedural correct rules for the dynamic reconfiguration of multilevel microsevice-based software architectures.

\section{THE COOPERATIVE CONTEXT}

From a modeling point of view, the different instances of a dynamic architecture describe both the different local compositions of a composite service and the different deployment configurations of a complex service interconnection. Taking into account the mutli-level factor in an architecture makes it possible to manage the end-to-end consistency between the different communication levels composing this architecture, and between the different nodes supporting its deployment. When adaptation affects transport level communication protocols, the deployment nodes correspond to the host machines and terminals. When it acts on the protocols of the lower levels of communication, a management of the configurations of the intermediary routers is necessary. When the adaptation acts on the clusters, the deployment nodes correspond to virtual machines. When it acts on services, a scale-up scale-down action of application-level clusters is necessary to manage the load increase/decrease. From an operational point of view, reconfiguration solutions work by adapting coordinated architectural transformations between the different communication levels of an architecture, and between the different nodes supporting its deployment.

This makes it possible to respond in a reactive or predictive way to changes in the functional requirements (e.g. the objectives of the mission of mobile cooperative robots) on the other hand, and to variations in the non-functional constraints related to quality of service and reliability (Fig. 2). Our approach constitutes an important step towards the automation of adaptive interactive distributed services necessary for autonomous cooperative communication. It deals, on the one hand, with the definition of compositional design methods supporting refinement and based on formal operational models for process automation engineering of complex or composite service architectures (SOA and SCA models). On the other hand, it deals with the design and implementation of software environments supporting dynamically reconfigurable architectural deployments.

Our approach and its applications cover different fields of technology and different fields of activities that can greatly influence the deployment and use of networks, and the software that uses and exploits them. The cooperative context of applications is both a feasibility hypothesis for solutions for the management of reconfiguration and QoS, and a source of complexity related to the associated high degree of dynamism and interaction. The cooperative context involves group communications or individual communications but also collective interest in resources sharing that influences the provisioning of the QoS [6]. This is in contrast to individual QoS requirements in any Internet-like context (as a global network and not as a than technology).
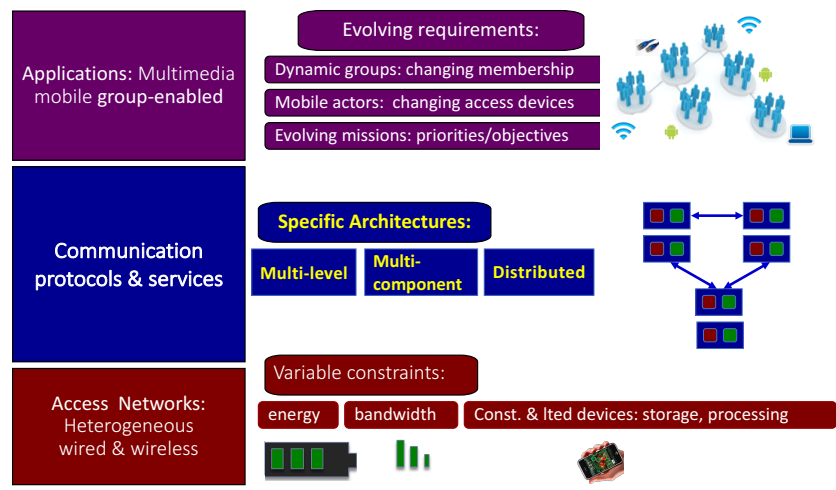

Figure 2: Multi-component layered architectures

The problem of maintaining quality of service is an important issue because it guarantees the usability of services. It is also difficult because it must have an end-to-end management without discontinuity, on the one hand at the different interaction levels of the distributed communication system, support for cooperation, and on the other hand during the various phases of the provisioning of a service (description, discovery, deployment and management). The problem of autonomy is the main challenge we address through dynamic reconfiguration. We have implemented different proofs of concepts as part of our research work in this domain mainly for the upper levels of communication. The challenge here is to generalize and extend this work to different levels of communication. Mastering the complexity of reconfiguration solutions is a challenge that we address by the formal compositional design and the multi-scale modeling [8]. Scaling up is another challenge that is best addressed by the rule-oriented characterization and the multi-scale modeling approach in general and our technique of management of dynamic architectures by of graph grammars, more particularly [7]. The policies we can implement do not require the enumeration of different architectural configurations and can be applied on abstract or refined models.

\section{THE APPROACH}

On the one hand, the approach explored is based on the adaptation of the communication and the cooperation protocols to network constraints and application requirements. On the other hand, it is based on the reconfiguration of system (e.g. microservice composition) at the different levels of the deployment architecture. The transformation of structures according to a semantics of graph grammars [7] is the technique underlying the management of dynamic architectures in our approach. For example, reconfiguration makes it possible to adapt the composition and distribution of components, services, microservices or partitions to network constraints, or to adapt communication channels to user mobility and changes in cooperation groups or shared spaces, or to changes in the priorities associated with the data exchanged.

Handling reconfiguration raises, at the same time, prob- 
lems relating to constraints and structural requirements (architectures: compositions, replications and distribution, and topologies), and problems related to constraints and requirements (functions and protocols). The associated methods cover both description and monitoring of quality of service parameters, and techniques for designing and managing cooperative communications. We investigate two main research directions that cover self-adaptability through configurable protocols, dynamic architectures, and associated coordination policies.

The first direction, namedDynamic architectures and selfconfigurable protocols, adresses the following three axis:

- the development of dynamically reconfigurable communication protocols to provide the best QoS that is, on the one hand, compatible with the nature of the exchanged information and the relative priorities of the messages and the actors, and on the other hand with the performance of the network and the collective objective of cooperation;

- the development of dynamically reconfigurable cooperation protocols for the management of spontaneous or predefined cooperative sessions. This includes the dynamic discovery of partners and services in distributed architectures (web services, microservices), session management and data sharing;

- the design of dynamic architectures both within a given protocol layer and between several layers, either locally or in a distributed way.

The second direction addresses Coordination policies for reconfiguration. It focuses on the development of adaptability policies for layers communication and cooperation, according to a model-oriented coordination approach. These policies are guided by the application requirements and the constraints of the network, and act on the one hand on the (re-)configuration of protocols, and on the transformation of architectures (dynamic deployment of components and / or microservice-based protocols of cooperation and communication).

\subsection{Foundations and objectives}

Our approach is based on the deployment and execution, validation, design support frameworks, and the concepts and the underlying modeling techniques resulting from our recent research on methods design and modeling for communication systems. Different techniques are integrated to transform and refine architectures and validate their conformity to styles in a doubly dynamic context for styles and architectures and at different levels of interaction, with local and distributed coordination. Graph grammars are adopted for characterization of architectural styles and for inter-level refinement, graph transformation for intra-level adaptation, and as visual notations for understanding and verification of reconfiguration.

Our work could extend and exploit the new theories for modeling autonomous interactive mobile and ubiquitous interactive systems. The recent calculus named "Bigraphs" by R. Milner is the reference theoretical framework of algebraic formal techniques, centered on interaction [9]. This calculus unifies approaches based on process algebra and graph-based approaches. It is a promising theory for research activities on the modeling of mobile and ubiquitous mobile interactive systems [1].

New communication systems are now implementing cooperative policies for solving problems of connectivity, reliability and quality of service. This is the case both for communication technologies of the type of mesh networks as well as for routing and data transport networks and in adhoc networks. Indeed, antennas or communication nodes can act as a relay to communicate information that is not intended for its users. Of the same way, several transport-level connections can cooperate to regularize the flow of information in a group of connections shared by applications on the same machine, or, by one or by a group of applications distributed on several clusters. The model of information producers and consumers, supported cooperatively by intermediaries for communication, is becoming widespread at the various communication levels after having proved itself in the middleware, session and application layers.

Both the theoretical foundations and the technical objectives make our research work is of interest for different collaborative projects we have participated in. Dynamic architectures, as support for reconfiguration and model-driven autonomy, and their application for communication and cooperation constitute one of the common problems to these projects, for different purposes and applications. The scope is important and covers a wide spectrum of use in different fields and for different applications with high autonomy requirements. This is particularly the case for embedded communication systems that constitute for us an area of investigation for the coming years with an application to the systems communicators and embedded software for the field of autonomous connected vehicles.

\subsection{Implementation}

Our objective is to cover the lower and upper architectural levels and to design new protocols based on microservice architectures. This would allow cooperative communication to be exploited to manage the adaptability of communication services. This allows us to answer both the problems of variation in communication resources (more and more present in the new adhoc and sensor networks) than to the problems of evolution in the application requirements.

For this purpose, different models and solutions need to be elaborated (Fig. 3). This includes: Resources Monitoring \& Analysis, Provisioning Planning \& Optimization, Adaptive Microservice-based communication protocols, Dynamic Topologies \& Architectures, and Cloud-native Algorithms \& Applications.

We designed and implemented an analytical framework for monitoring and analyzing QoS of publish/subscribe systems on MANET. Our framework copes with intermittent connectivity frequently occurring in MANET and provides statistical methods allowing detecting QoS degradations affecting links between brokers at the middleware layer [5]. We designed and implemented a model driven methodology that enables the dynamic reconfiguration by generating autonomic architectures from high level descriptions of functional requirements [3]. We have implemented autonomic communication protocols [11] based on micro-protocols.

We have designed and implemented a collaborative framework for communications in M2M wireless networks. This framework is composed of a context-aware and applicationaware software platform and a reasoning machine for net- 


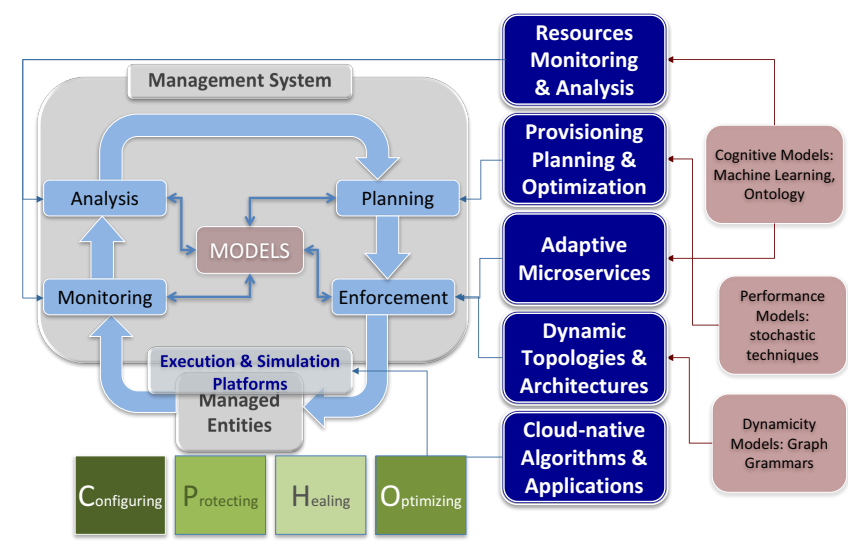

Figure 3: Autonomic Properties, Models, and entities

work management. The software platform uses multi-level adaptation mechanisms to support dynamic collaboration activities [10].

We have designed a Dynamically Reconfigurable Architecture for Autonomic Services able to provide autonomic properties for QoS management in web service-based distributed applications. This architecture has been implemented and experimented successfully with different use cases. It covers the whole cycle of autonomic management including monitoring and analysis of QoS parameters, planning and execution [4].

Our implemented solutions can be viewed as a set of services constituting part of the so-called Autonomic Service Bus (ASB) as described in Fig. 4 and that we can imagine as an integration solution for the discussed issues.

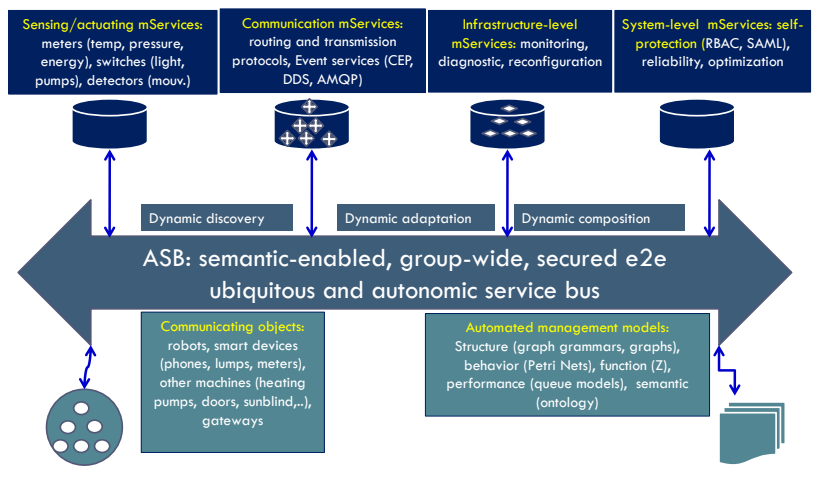

Figure 4: Autonomic Service Bus architecture

\section{CONCLUSIONS}

Our research work contributes to model-driven design and management of cooperative distributed communicative systems, complex and critical resilient autonomous systems. We elaborated different description approaches for dynamically reconfigurable software architectures as well as policyoriented and rule-based management of communication networks and distributed ubiquitous interactive systems. Important theoretical work has been achieved on the problem of modeling of Systems of Systems.

\section{ACKNOWLEDGMENTS}

The author would like to thank all his collaborators as well as the COMU-IDEX project of Univ. de Toulouse that has partially funded this work.

\section{REFERENCES}

[1] A.Gassara, I.Bouassida, M.Jmaiel, and K.Drira. A bigraphical multi-scale modeling methodology for system of systems. Computers and Electrical Engineering, 58:113-125, Feb 2017.

[2] M. Alaya, S.Medjiah, T.Monteil, and K.Drira. Towards semantic data interoperability in onem $2 \mathrm{~m}$ standard. IEEE Communications Magazine, 53(12):35-41, Dec 2015.

[3] E.Mezghani, R. Halima, I.Bouassida, and K.Drira. A model driven methodology for enabling autonomic reconfiguration of service oriented architecture. In Annual ACM Symposium on Applied Computing, pages -2, Mar 2013.

[4] E.Mezghani, R. Halima, and K.Drira. DRAAS: Dynamically Reconfigurable Architecture for Autonomic Services, pages 483-505. Springer, Jan 2014.

[5] I.Abdennadher-Lahyani, L. Amor, M.Jmaiel, K.Drira, and C.Chassot. Analytical framework for qos aware publish/subscribe system deployed on manet. In IEEE International Symposium on Parallel and Distributed Processing with Applications (ISPA 2012), pages -7, Jul 2012.

[6] I.Bouassida, K.Drira, C.Chassot, and M.Jmaiel. A model-based multi-level architectural reconfiguration applied to adaptability management in context-aware cooperative communication support systems. In Working IEEE/IFIP Conference on Software Architecture 86 European Conference on Software Architecture, WICSA/ECSA 2009, pages 353-356, Sep 2009.

[7] I.Bouassida, K.Guennoun, K.Drira, C.Chassot, and M.Jmaiel. A rule-driven approach for architectural self adaptation in collaborative activities using graph grammars. International Journal of Autonomic Computing, 1(3):226-245, Mar 2010.

[8] I.Khlif, M. Kacem, A. Kacem, and K.Drira. Towards a multi-scale modeling approach for software architectures. In Annual ACM Symposium on Applied Computing, pages -4, Apr 2015.

[9] R. Milner. Bigraphical reactive systems. In K. G. Larsen and M. Nielsen, editors, CONCUR 2001 Concurrency Theory, 12th International Conference, Aalborg, Denmark, August 20-25, 2001, Proceedings, volume 2154 of Lecture Notes in Computer Science, pages 16-35. Springer, 2001.

[10] A. Mougy, A.Kamoun, M.Ibnkahla, S.Tazi, and K.Drira. A context and application-aware framework for resource management in dynamic collaborative wireless $\mathrm{m} 2 \mathrm{~m}$ networks. Journal of network and computer applications, 44:30-45, Sep 2014.

[11] N. Wambeke, F.Armando, K.Guennoun, K.Drira, E.Exposito, and C.Chassot. Towards the use of models for autonomic network management. In IFIP Joint Conference on Personal Wireless Communications (PWC'2008), pages 459-470, Sep 2008. 